\title{
Prospective and detailed behavioral phenotyping in DDX3X syndrome
}



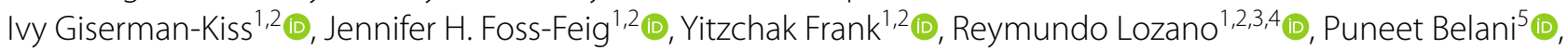

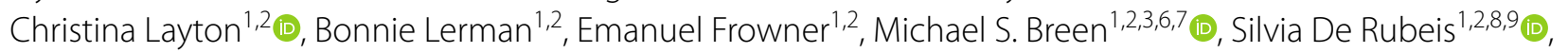

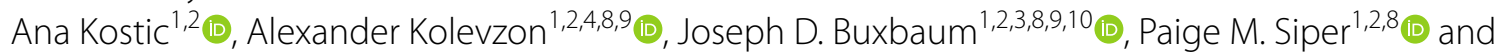 \\ Dorothy E. Grice ${ }^{1,2,8,9,11^{*}}$ (1)
}

\begin{abstract}
Background: DDX3X syndrome is a recently identified genetic disorder that accounts for $1-3 \%$ of cases of unexplained developmental delay and/or intellectual disability (ID) in females, and is associated with motor and language delays, and autism spectrum disorder (ASD). To date, the published phenotypic characterization of this syndrome has primarily relied on medical record review; in addition, the behavioral dimensions of the syndrome have not been fully explored.
\end{abstract}

Methods: We carried out multi-day, prospective, detailed phenotyping of DDX3X syndrome in 14 females and 1 male, focusing on behavioral, psychological, and neurological measures. Three participants in this cohort were previously reported with limited phenotype information and were re-evaluated for this study. We compared results against population norms and contrasted phenotypes between individuals harboring either (1) protein-truncating variants or (2) missense variants or in-frame deletions.

Results: Eighty percent (80\%) of individuals met criteria for ID, 60\% for ASD and 53\% for attention-deficit/hyperactivity disorder (ADHD). Motor and language delays were common as were sensory processing abnormalities. The cohort included 5 missense, 3 intronic/splice-site, 2 nonsense, 2 frameshift, 2 in-frame deletions, and one initiation codon variant. Genotype-phenotype correlations indicated that, on average, missense variants/in-frame deletions were associated with more severe language, motor, and adaptive deficits in comparison to protein-truncating variants.

Limitations: Sample size is modest, however, DDX3X syndrome is a rare and underdiagnosed disorder.

Conclusion: This study, representing a first, prospective, detailed characterization of DDX3X syndrome, extends our understanding of the neurobehavioral phenotype. Gold-standard diagnostic approaches demonstrated high rates of ID, ASD, and ADHD. In addition, sensory deficits were observed to be a key part of the syndrome. Even with a modest sample, we observe evidence for genotype-phenotype correlations with missense variants/in-frame deletions generally associated with more severe phenotypes.

Keywords: Autism, Developmental delay, Genotype-phenotype correlation, Intellectual disability, DDX3X syndrome

*Correspondence: Dorothy.Grice@mssm.edu

†Lara Tang and Tess Levy have contributed equally to this work

1 Seaver Autism Center for Research and Treatment, Icahn School

of Medicine at Mount Sinai, 1425 Madison Avenue, Box 1230, New York, NY 10029, USA

Full list of author information is available at the end of the article

\section{Background}

A significant proportion of ASD and associated neurodevelopmental disorders (NDDs), including ID, global developmental delay (DD) and epilepsy, are linked to ultrarare genetic variants. As exome and genome 
sequencing become more accessible, many novel genetic NDD syndromes are being identified. These include DDX3X syndrome, which is emerging as one of the most common genetic causes of ID/DD in females $[1,2]$ and is also significantly associated with ASD [3-6].

The $D D X 3 X$ gene encodes a ubiquitously expressed ATP-dependent DEAD-box RNA helicase, involved in mRNA biogenesis, RNA metabolism, and mRNA translation $[7,8]$. Recently, it has been shown that DDX3X is involved in the anti-viral innate immune response, stress granule nucleation and localization, apoptotic signaling following DNA damage, maintenance of lipid homeostasis, cell cycle control, and regulation of Wnt- $\beta$-catenin signaling [8-11]. In the central nervous system, DDX3X is essential for neurite outgrowth and synaptogenesis, and for the proliferation and differentiation of cortical neural progenitors $[12,13]$.

In the large national Deciphering Developmental Disabilities study, which serially ascertained participants with $\mathrm{DD}$, investigators found high rates of $D D X 3 X$ variants $\left(P<10^{-50}\right)$ [2]. Similarly, a recent large-scale sequencing study of participants ascertained for ASD identified $D D X 3 X$ as a genome-wide significant ASD gene [3], and multiple exome studies also identified $D D X 3 X$ variants in ASD cohorts $[3,5,6,14]$. Finally, studies of cohorts with $D D X 3 X$ variants demonstrated high rates of developmental delays or intellectual disability ( 50-100\%), structural brain changes (25-90\%) - most commonly, abnormalities of the corpus callosum (25-87\%) and cortical dysplasias
(11-12\%) - and behavioral abnormalities, including ASD, hyperactivity, and aggression (21-53\% combined) [1, 13, $15,16]$. However, these phenotypic studies relied almost exclusively on retrospective data analyses.

$D D X 3 X$ is located in a short region of $\mathrm{Xp} 11.4$ that escapes X-inactivation [17, 18], thus females with the syndrome are functionally heterozygous for deleterious variants. Most of the identified $D D X 3 X$ variants are in females; it is assumed that males carrying a very deleterious $D D X 3 X$ variant do not survive to term, a finding confirmed in mouse models [17]. Surviving male patients carry missense variants that are thought to act as hypomorphic alleles $[19,20]$. Because $D D X 3 X$ variants appear to be very penetrant, all variants that have been identified in females to date are de novo.

In the current study, we carried out comprehensive, inperson, prospective phenotyping of 15 participants with DDX3X syndrome, focusing on neurobehavioral outcomes. We compared all normed assessments to population norms, and compared phenotypes in participants with protein-truncating variants versus other variants.

\section{Methods}

Fifteen individuals (14 female; 1 male) from 3 to 16 years old (7.5 \pm 4.5 years) diagnosed with DDX3X syndrome were evaluated at the Seaver Autism Center at the Icahn School of Medicine at Mount Sinai (Fig. 1). Three participants have been previously reported (Additional file 1: Table S1). All participants were seen for a 3-4 day

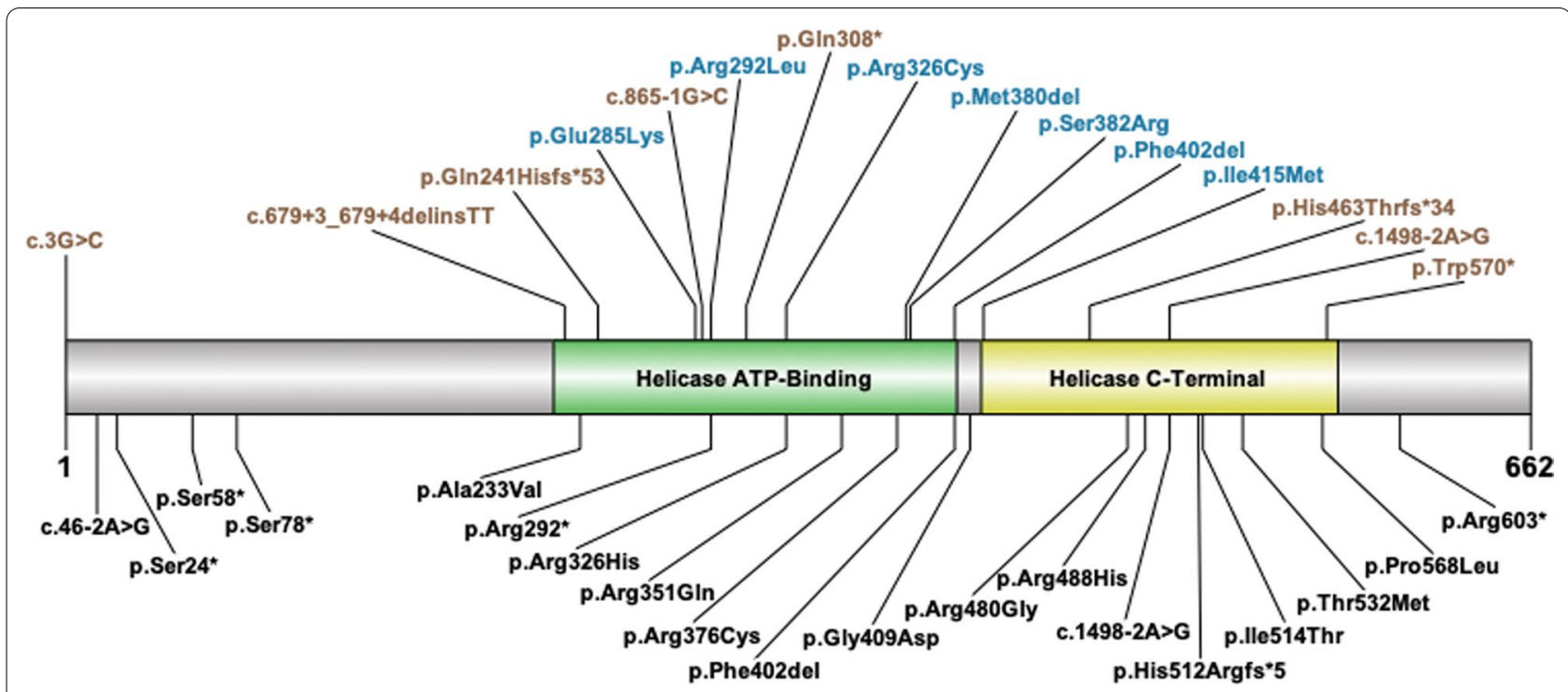

Fig. 1 DDX3X variants. Top, variants in the cohort: protein-truncating variants (PTVs) are colored tan, while missense variants and in-frame deletions are colored blue. The male participant carries the p.Arg292Leu variant. Bottom, recurrent variants: variants reported at least three times in the literature and/or in ClinVar. The helicase ATP-binding and helicase C-terminal domains are shown as reported in Uniprot O00571 
in-person visit at the Seaver Autism Center, including approximately $9 \mathrm{~h}$ of direct assessment, $6 \mathrm{~h}$ of caregiver interviews, and $4 \mathrm{~h}$ of caregiver questionnaires (Table 1 ). All neuropsychological testing was completed by research-reliable clinical psychologists. Medical evaluations (psychiatric, neurologic, and clinical genetic) were completed by board-certified clinicians. In addition, a board-certified radiologist, with specialty training in neuroradiology, reviewed all available MRI scans. All other available medical records were reviewed by the study lead. This study was approved by the Mount Sinai Institutional Review Board. Legal guardians of each participant gave informed consent prior to study participation and additional consent prior to publication.

\section{Genetic testing}

Variants were identified and validated at Clinical Laboratory Improvement Amendments certified laboratories, as was de novo status. Variants were reannotated according to the Human Genome Variation Society Guidelines, with nucleotide and amino acid positions mapped to the DDX3X RefSeq transcript NM_001356.4, and interpreted using the American College of Medical Genetics and Genomics and Association for Molecular Pathology Guidelines [21] (Additional file 1: Table S1). Variants were annotated as either (1) protein-truncating variants (PTVs, including nonsense, frameshift, splice-site and start-codon loss variants), or (2) missense or in-frame deletions.

\section{Intellectual and adaptive function}

Intellectual functioning was assessed by clinical psychologists using the Stanford-Binet Intelligence Scales, 5th Edition [22], the Differential Abilities Scales, 2nd Edition (DAS-II) [23], or the Mullen Scales of Early Learning [24]. Full scale Intelligence Quotient (IQ), nonverbal IQ, and verbal IQ scores were calculated for those participants who completed the Stanford-Binet- 5 or the DASII. Developmental quotient (DQ) scores were calculated from age equivalents for all participants and used for a cohort-wide comparison of cognitive abilities. Adaptive functioning was evaluated using the Vineland Adaptive Behavior Scales, $3^{\text {rd }}$ Edition (Vineland-3) [25], which was

Table 1 Study approach

\begin{tabular}{|c|c|}
\hline Domain & Measure \\
\hline Intellectual and Adaptive Functioning & $\begin{array}{l}\text { Stanford-Binet Intelligence Scales, Fifth Edition } \\
\text { Differential Abilities Scales, Second Edition (DAS-II) } \\
\text { Mullen Scales of Early Learning } \\
\text { Vineland Adaptive Behavior Scales, Third Edition (Vineland-3) }\end{array}$ \\
\hline Motor Functioning & $\begin{array}{l}\text { Autism Diagnostic Interview-Revised (ADI-R) subscales, ADI Regres- } \\
\text { sion supplement } \\
\text { Clinical evaluation by a psychiatrist } \\
\text { Vineland-3 } \\
\text { Beery-Buktenica Developmental Test of Visual-Motor Integration, } \\
\text { Sixth Edition (VMI-6) } \\
\text { Developmental Coordination Disorder Questionnaire (DCDQ) }\end{array}$ \\
\hline Expressive and Receptive Language & $\begin{array}{l}\text { ADI-R subscales } \\
\text { Expressive Vocabulary Test, Second Edition } \\
\text { Peabody Picture Vocabulary Test, Fourth Edition } \\
\text { Vineland-3 } \\
\text { MacArthur-Bates Communicative Development Inventory }\end{array}$ \\
\hline ASD Symptomatology & $\begin{array}{l}\text { Clinical evaluation by a psychiatrist } \\
\text { ADI-R } \\
\text { Autism Diagnostic Observation Schedule, Second Edition (ADOS-2) } \\
\text { Social Responsiveness Scale, Second Edition (SRS-2) } \\
\text { Repetitive Behavior Scale-Revised (RBS-R) }\end{array}$ \\
\hline Sensory Features & $\begin{array}{l}\text { Sensory Assessment for Neurodevelopmental Disorders (SAND) } \\
\text { Short Sensory Profile (SSP) }\end{array}$ \\
\hline Behavioral Comorbidities & $\begin{array}{l}\text { Achenbach Child Behavior Checklist (CBCL) } \\
\text { Aberrant Behavior Checklist } \\
\text { Vineland-3 } \\
\text { Clinical evaluation by a psychiatrist }\end{array}$ \\
\hline Medical Evaluation & $\begin{array}{l}\text { Medical and psychiatric history/evaluation } \\
\text { Neurological exam } \\
\text { Dysmorphology exam } \\
\text { Review of neuroimaging } \\
\text { Pervasive Developmental Disorder Behavior Inventory }\end{array}$ \\
\hline
\end{tabular}


administered by clinical psychologists. ID was diagnosed based on the Diagnostic and Statistical Manual of Mental Disorders, Fifth Edition (DSM-5) criteria [26].

\section{Motor function}

Major motor milestones were evaluated using the Autism Diagnostic Interview-Revised (ADI-R) [27] and ADI regression supplement [28], administered by clinical psychologists, and psychiatric evaluation by a child and adolescent psychiatrist. Fine and gross motor skills were evaluated using the Vineland- 3 and Mullen subscales. To assess the integration of skills in the visualmotor domain, clinical psychologists administered the Beery-Buktenica Developmental Test of Visual-Motor Integration, Sixth Edition (VMI-6) [29]. Caregivers completed the Developmental Coordination Disorder Questionnaire (DCDQ) [30].

\section{Expressive and receptive language}

Language milestones were obtained using the ADI-R and the ADI regression supplement. Direct assessment of expressive language was assessed by the Expressive Vocabulary Test, Second Edition [31], and receptive language by the Peabody Picture Vocabulary Test, Fourth Edition [32], both administered by clinical psychologists. The language subdomains of the Vineland-3 and Mullen Scales were also analyzed. Caregivers completed the MacArthur-Bates Communicative Development Inventory [33].

\section{ASD symptomatology and sensory features}

Consensus DSM-5 [26] ASD diagnoses were determined from the psychiatric evaluation and results from gold-standard diagnostic assessments including the Autism Diagnostic Observation Schedule, Second Edition (ADOS-2) [34], and the ADI-R [27]. Caregiver questionnaires, including the Social Responsiveness Scale, Second Edition (SRS-2) [35] and the Repetitive Behavior Scale-Revised (RBS-R) [36], were used to further evaluate ASD features.

Sensory symptoms were assessed using the Sensory Assessment for Neurodevelopmental Disorders (SAND) [37], administered by a clinical psychologist, and the Short Sensory Profile (SSP) [38], a caregiver questionnaire.

\section{Behavioral comorbidities}

The Achenbach Child Behavior Checklist (CBCL) [39] and the Aberrant Behavior Checklist [40-43] were used to assess additional psychiatric features and behavioral challenges. The Vineland-3 Maladaptive Behavior domain and subdomains of Internalizing and Externalizing Behavior were evaluated. Other behavioral and psychiatric comorbidities were documented during the psychiatric evaluation.

\section{Medical evaluation}

A neurological examination was completed by a pediatric neurologist. The examination assessed motor and sensory skills, balance and coordination, mental status, and reflexes. Dysmorphic features were assessed by a clinical geneticist. Medical history was assessed by parent report and review of medical records by the study psychiatrist. Brain MRI scans $(n=12)$ or clinical reports $(n=2)$ were reviewed by the study neuroradiologist, and clinically significant findings were documented. Caregivers completed the Pervasive Developmental Disorder Behavior Inventory [44] to supplement characterization of sleep disturbance and prenatal and neonatal complications.

\section{Results}

All $D D X 3 X$ variants were classified as pathogenic or likely pathogenic. Variants included 5 missense variants, 3 intronic/splice-site variants, 2 nonsense variants, 2 frameshift variants, 2 in-frame deletions, and one initiation codon variant (Fig. 1, Additional file 1: Table S1). The variants clustered in the helicase ATP-Binding and helicase C-Terminal domains. De novo status was confirmed in $14 / 15$ individuals, and the remaining individual had maternal inheritance ruled out. The male participant carried a de novo missense variant.

\section{Intellectual and adaptive functioning}

Nine of the 15 participants in the cohort completed the Mullen Scales, 5 completed the Stanford-Binet-5, and 1 was administered the DAS-II. Standard scores (population mean $=100, \mathrm{SD}=15$ ) across the 6 individuals for which IQs could be calculated ranged from 40 to 85 $(58.7 \pm 17.2)$ for full scale IQ, 42 to $87(59.7 \pm 16.1)$ for nonverbal IQ, and 43 to $111(65.7 \pm 25.6)$ for verbal IQ. Full scale DQ, nonverbal DQ, and verbal DQ were calculated for all participants: 14/15 participants showed greater than a $40 \%$ delay in all scores; one participant showed average scores for full scale DQ, nonverbal $\mathrm{DQ}$, and an above average score in verbal DQ (Table 2, Fig. 2a). On the Vineland-3, average standard scores for Communication, Daily Living Skills, Socialization, and the Adaptive Behavior Composite were 3 to 4 standard deviations below the general population mean (Table 2, Fig. 2b, Additional file 1: Table S2). Overall, 12 of the 15 participants in this cohort were given a diagnosis of ID based on DSM-5 criteria. 
Table 2 Summary statistics for clinical measures

\begin{tabular}{|c|c|c|c|c|c|c|c|}
\hline Domain & Evaluation & Variable & $\mathbf{n}$ & Range & Mean & SD & $\begin{array}{l}\% \text { with } \\
\text { atypical } \\
\text { score }\end{array}$ \\
\hline \multirow{7}{*}{$\begin{array}{l}\text { Intellectual and Adaptive Func- } \\
\text { tioning }\end{array}$} & \multirow{3}{*}{$\begin{array}{l}\text { Mullen Scales, Stanford- } \\
\text { Binet-5, DAS-II }\end{array}$} & Full scale DQ & 15 & $9.9-97.8$ & 34.1 & 22.5 & 93 \\
\hline & & Nonverbal DQ & 15 & $14.1-78.4$ & 34.1 & 16.8 & 93 \\
\hline & & Verbal DQ & 15 & $5.8-117.3$ & 35.1 & 28.7 & 93 \\
\hline & \multirow[t]{4}{*}{ Vineland- $3^{2}$} & Communication & 15 & $20-87$ & 43.7 & 24.7 & 87 \\
\hline & & Daily Living Skills & 15 & $20-73$ & 48.6 & 17.5 & 100 \\
\hline & & Socialization & 15 & $20-90$ & 55.3 & 20.6 & 87 \\
\hline & & Adaptive Behavior Composite & 15 & $20-76$ & 49.5 & 18.8 & 100 \\
\hline \multirow[t]{7}{*}{ Motor Functioning } & \multirow[t]{2}{*}{ ADI-R, Psychiatric Evaluation² } & Age of crawling (months) & 15 & $5-24$ & 15.2 & 6.0 & 87 \\
\hline & & $\begin{array}{l}\text { Age of walking independently } \\
\text { (months) }\end{array}$ & 13 & $11-36$ & 26 & 8.1 & 93 \\
\hline & \multirow[t]{3}{*}{ Vineland- $3^{2}$} & Motor Skills & 10 & $20-77$ & 57.6 & 18.6 & 100 \\
\hline & & Fine Motor & 10 & $1-10$ & 6.0 & 3.7 & 100 \\
\hline & & Gross Motor & 10 & $1-12$ & 7.9 & 4.3 & 60 \\
\hline & VMI- $6^{3}$ & Standard score & 13 & $<45-87$ & 68.3 & 10.0 & 85 \\
\hline & $\mathrm{DCDQ}^{4}$ & Total score & 8 & $15-45$ & 23.8 & 9.7 & 100 \\
\hline \multirow{11}{*}{$\begin{array}{l}\text { Expressive and Receptive Lan- } \\
\text { guage }\end{array}$} & \multirow[t]{2}{*}{ ADI-R, Psychiatric Evaluation² } & Age of first word (months) & 10 & $15-60$ & 28.7 & 14.0 & 87 \\
\hline & & Age of phrased speech (months) & 7 & $36-60$ & 48.6 & 9.8 & 100 \\
\hline & Expressive Vocabulary Test ${ }^{2}$ & Standard score & 7 & $44-123$ & 77.6 & 23.5 & 57 \\
\hline & $\begin{array}{l}\text { Peabody Picture Vocabulary } \\
\text { Test }^{2}\end{array}$ & Standard score & 13 & $20-111$ & 59.7 & 31.2 & 69 \\
\hline & \multirow[t]{2}{*}{ Vineland- $3^{2}$} & Expressive Language & 15 & $1-14$ & 3.6 & 4.3 & 93 \\
\hline & & Receptive Language & 15 & $1-14$ & 5.7 & 4.8 & 80 \\
\hline & \multirow{5}{*}{$\begin{array}{l}\text { MacArthur-Bates Communica- } \\
\text { tive Development Inventory }\end{array}$} & Words Understood & 13 & 15-396 & 277.9 & 125.8 & $\mathrm{n} / \mathrm{a}$ \\
\hline & & Words Produced & 13 & $0-396$ & 164.8 & 186.1 & $\mathrm{n} / \mathrm{a}$ \\
\hline & & Early Gestures & 13 & $6-18$ & 12.7 & 4.9 & $\mathrm{n} / \mathrm{a}$ \\
\hline & & Later Gestures & 13 & $4-45$ & 27.2 & 15.4 & $\mathrm{n} / \mathrm{a}$ \\
\hline & & Total Gestures & 13 & $10-63$ & 39.9 & 19.5 & $\mathrm{n} / \mathrm{a}$ \\
\hline \multirow[t]{8}{*}{ ASD Symptomatology } & \multirow[t]{6}{*}{$\mathrm{SRS}-2^{5}$} & Total score & 15 & $50-96$ & 68.9 & 14.0 & 73 \\
\hline & & Social Communication & 15 & $48-94$ & 69.7 & 16.4 & 60 \\
\hline & & Social Awareness & 15 & $56-90$ & 70.4 & 10.6 & 80 \\
\hline & & Social Cognition & 15 & $51-84$ & 66.8 & 10.7 & 80 \\
\hline & & Repetitive Behavior & 15 & $52-90$ & 72.6 & 13.5 & 80 \\
\hline & & Social Motivation & 15 & $38-86$ & 58.2 & 14.1 & 47 \\
\hline & \multirow[t]{2}{*}{ RBS-R } & Insistence on Sameness & 14 & $0-9$ & 2.57 & 2.93 & 3 n/a \\
\hline & & Stereotyped Behavior & 14 & $0-6$ & 2.64 & 2.02 & $2 n / a$ \\
\hline \multirow[t]{6}{*}{ Sensory Features } & \multirow[t]{6}{*}{ SAND $^{2}$} & Sensory seeking & 15 & $7-28$ & 17.27 & 7.3 & 87 \\
\hline & & Hyporeactivity & 15 & $0-21$ & 10.40 & 6.7 & 80 \\
\hline & & Hyperreactivity & 15 & $2-11$ & 5.67 & 3.5 & 47 \\
\hline & & Tactile & 15 & $2-19$ & 13.67 & 4.5 & 93 \\
\hline & & Auditory & 15 & $0-23$ & 10.6 & 6.3 & 73 \\
\hline & & Visual & 15 & $0-17$ & 9.07 & 5.5 & 73 \\
\hline
\end{tabular}


Table 2 (continued)

\begin{tabular}{|c|c|c|c|c|c|c|c|}
\hline Domain & Evaluation & Variable & $\mathrm{n}$ & Range & Mean & SD & $\begin{array}{l}\% \text { with } \\
\text { atypical } \\
\text { score }\end{array}$ \\
\hline \multirow[t]{13}{*}{ Behavioral Comorbidities } & \multirow[t]{6}{*}{$\mathrm{CBCL}^{6}$} & Internalizing & 14 & $33-69$ & 53.50 & 9.51 & 114 \\
\hline & & Depressive & 14 & $50-77$ & 58.86 & 8.42 & 221 \\
\hline & & Anxiety & 14 & $50-76$ & 54.71 & 7.06 & 614 \\
\hline & & Externalizing & 14 & $44-73$ & 58.43 & 8.11 & 121 \\
\hline & & $\mathrm{ADHD}$ & 14 & $54-72$ & 64.29 & 6.60 & 050 \\
\hline & & Oppositional/Defiant & 14 & $51-64$ & 54.71 & 5.30 & 00 \\
\hline & \multirow[t]{5}{*}{ Aberrant Behavior Checklist ${ }^{5}$} & Hyperactivity & 15 & $<40-65$ & 53.60 & 7.50 & 020 \\
\hline & & Irritability & 15 & $<40-67$ & 50.67 & 9.79 & 913 \\
\hline & & Lethargy/Social Withdrawal & 15 & $<40-60$ & 48.11 & 7.64 & 47 \\
\hline & & Stereotypy & 15 & $40-73$ & 49.53 & 10.14 & 420 \\
\hline & & Inappropriate Speech & 15 & $40-67$ & 48.93 & 8.03 & 37 \\
\hline & \multirow[t]{2}{*}{ Vineland- $3^{2}$} & Internalizing Behavior & 15 & $15-22$ & 18.5 & 2.2 & 33 \\
\hline & & Externalizing Behavior & 15 & $11-21$ & 17.5 & 2.6 & 20 \\
\hline
\end{tabular}

DAS-II: Differential Abilities Scales, Second Edition; ADI-R: Autism Diagnostic Interview-Revised; VMI-6: Beery-Buktenica Developmental Test of Visual-Motor Integration, Sixth Edition; DCDQ: Developmental Coordination Disorder Questionnaire; SRS-2: Social Responsiveness Scale, Second Edition; RBS-R: Repetitive Behavior Scales-Revised; SAND: Sensory Assessment for Neurodevelopmental Disorders; CBCL: Achenbach Child Behavior Checklist

${ }^{1}$ Atypical if $<77.5$

${ }^{2}$ Atypical if standard score 1.5 SD above or below population mean

${ }^{3}$ Atypical if standard score $<50$, per manual

${ }^{4}$ Atypical if score 15-46 (5 y-7 y $\left.11 \mathrm{~m}\right), 15-55$ (8 y-9 y $\left.11 \mathrm{~m}\right), 15-57$ (10 y-15 y)

${ }^{5}$ Atypical if T score $>60$

${ }^{6}$ Atypical if T score $>65$

\section{Motor functioning}

Delays in age of first crawling were reported in 13 of 15 participants (Table 2). Two of the participants, both 5 years old, could not walk unaided at the time of assessment, while the age of first walking in the 13 other participants ranged from 11 to 36 months.

Ten participants in the cohort were administered the Motor domain of the Vineland-3 (Fig. 2b, Additional file 1: Table S2). Average scores in the Motor Domain and Gross Motor subdomain were 3 standard deviations below the general population mean, while the Fine Motor subdomain scores were 4 standard deviations below the mean (Table 2). Based on the Vineland-3, 2 individuals had significantly better gross motor than fine motor abilities, while 1 showed better fine motor ability. On the Mullen Scales, 4 individuals showed better gross motor than fine motor abilities. However, overall, the cohort did not show significant differences between fine and gross motor abilities. On the VMI- $6(n=13,2$ attempted but could not complete), the average standard score of the cohort was 2 standard deviations below the general population mean, with 7 individuals scoring in the impaired range (Additional file 1: Table S2). On the DCDQ $(n=8)$, $100 \%$ of participants scored in a range indicative and/or suspect of a Developmental Coordination Disorder.

\section{Expressive and receptive language}

Early language milestones, collected from the ADI-R and psychiatric evaluation, were delayed for all 15 participants. At the time of assessment, 5 participants in the cohort were non-verbal (Fig. 3a). Of the 10 participants with verbal capacity, the age when first words emerged ranged from 15 to 60 months, and 7 individuals achieved phrase speech between 36 to 60 months (Table 2).

Expressive language, assessed by the Expressive Vocabulary Test, Second Edition, was completed by 7 participants; 8 did not have skills to achieve the base score. For those who completed the assessment, standard scores ranged from 44 to $123(77.6 \pm 23.5)$. Receptive language, assessed by the Peabody Picture Vocabulary Test, Fourth Edition, was completed by 13 participants, while 2 did not have the skills to achieve the base score; standard scores ranged from 20 to $111(59.7 \pm 31.2)$. On the Vineland-3, Expressive and Receptive Language subdomains 

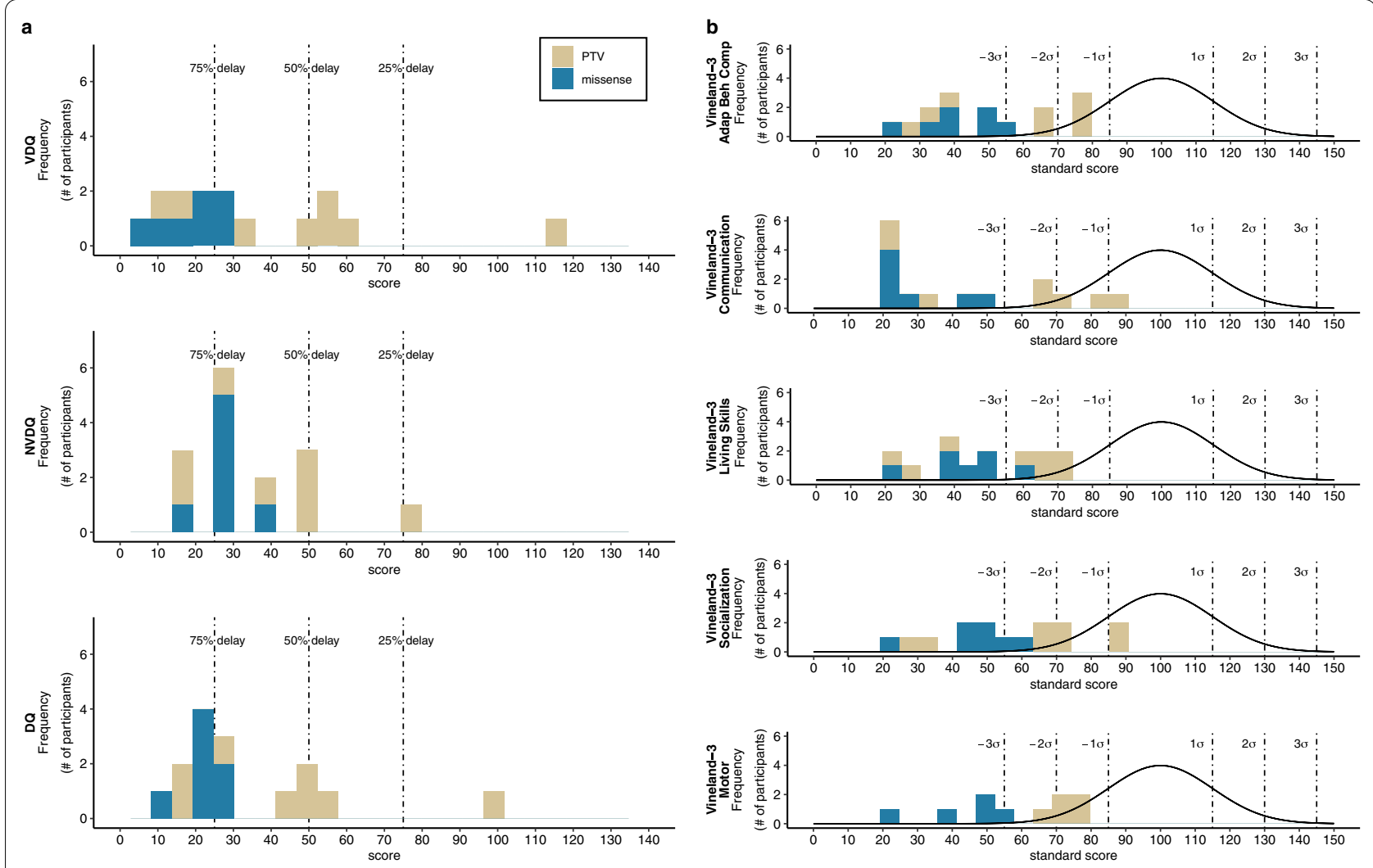

Fig. 2 Intellectual, adaptive and motor functioning. a Frequency histograms of verbal, nonverbal, and fullscale developmental quotients (VDQ, NVDQ, DQ). $\mathbf{b}$ Frequency histograms of standard scores on domains of the Vineland-3: Adaptive Behavior Composite, Communication, Daily Living Skills, Socialization, and Motor (the Maladaptive Behavior domain is represented in Additional file 2: Fig. S1B). All plots show frequency (i.e., number of individuals) in each bin. Developmental delays of $25 \%, 50 \%$, and $75 \%$ are indicated by dashed lines (a), while distribution of standard scores in typically developing individuals are shown as black lines $(\mathbf{b})$, together with associated standard deviations (dashed lines). PTV, protein-truncating variant; missense, missense variant or in-frame deletion

showed similar variability (Table 2). Receptive and expressive language was compared within individuals using the Expressive Vocabulary Test and Peabody Picture Vocabulary Test $(n=7)$ and the Vineland-3 subscales $(n=15)$. Using the Peabody Picture Vocabulary Test and Expressive Vocabulary Test, 2 individuals showed significantly higher expressive than receptive language ability, and 2 showed significantly higher receptive than expressive ability ( $>12$ difference in standard score). However, it is important to note that 4 individuals could complete the Peabody Picture Vocabulary Test but could not complete the Expressive Vocabulary Test. Using the Vineland-3, $47 \%$ showed significantly higher receptive than expressive language abilities.

On the MacArthur-Bates Communicative Development Inventory $(n=13)$, parents reported the average number of words understood was 278 and words produced was 165 (from of a total of 396 queried), again indicating higher receptive than expressive skills. On average, individuals had 13 early gestures (e.g., shakes head no, blows kiss), and 27 later gestures (e.g., brushes teeth, waters plants), with the average of total gestures being 40 (from a total of 63).

\section{ASD symptomatology}

Nine of 15 participants (60\%) received a consensus DSM-5 diagnosis of ASD based on clinical evaluation, ADOS-2, and ADI-R. Individuals in this cohort were administered ADOS-2 Module $1(n=10), 2(n=3)$, $3(n=1)$, and $4(n=1)$. Based on results from the ADOS-2 alone, $20 \%$ of participants met diagnostic criteria for an autism spectrum classification and 53.3\% for an autism classification (Fig. 3b, Additional file 1: Table S2). The ADOS-2 had 9 true positive results, 3 true negative results, and 3 false positive results, when compared to consensus diagnosis. False positives were present in all 3 participants who received an ADOS-2 Module 2, which is administered to individuals with phrase speech. A review of results from the ADOS-2, and cognitive and language assessments, 

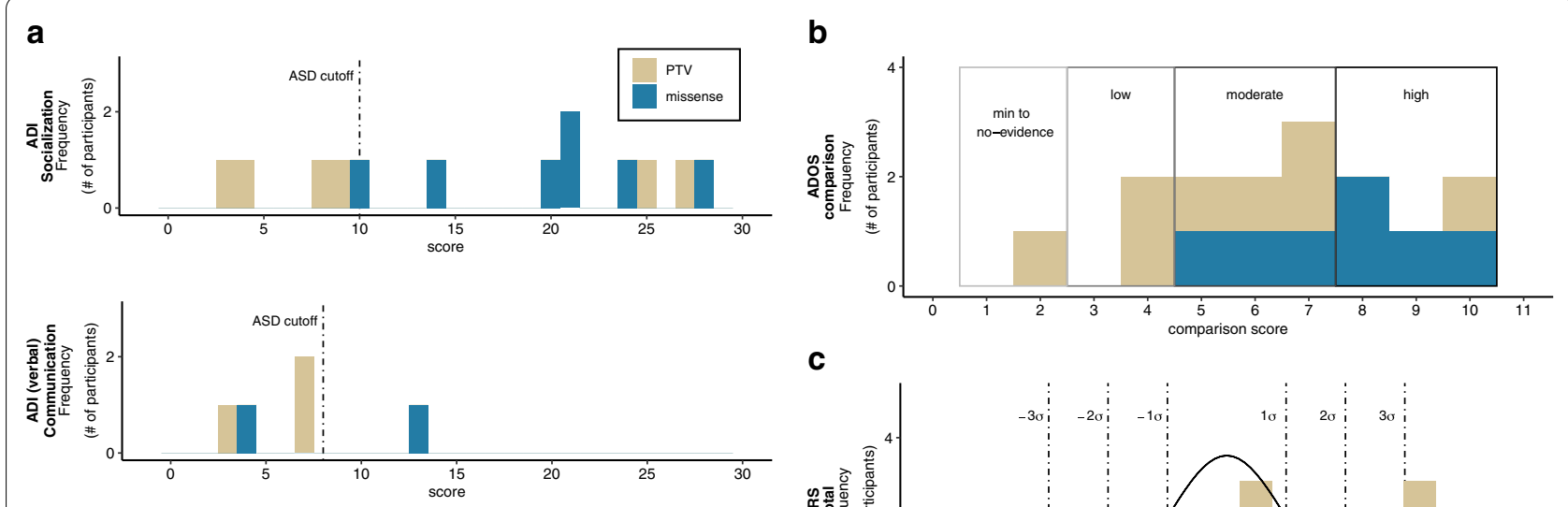

C
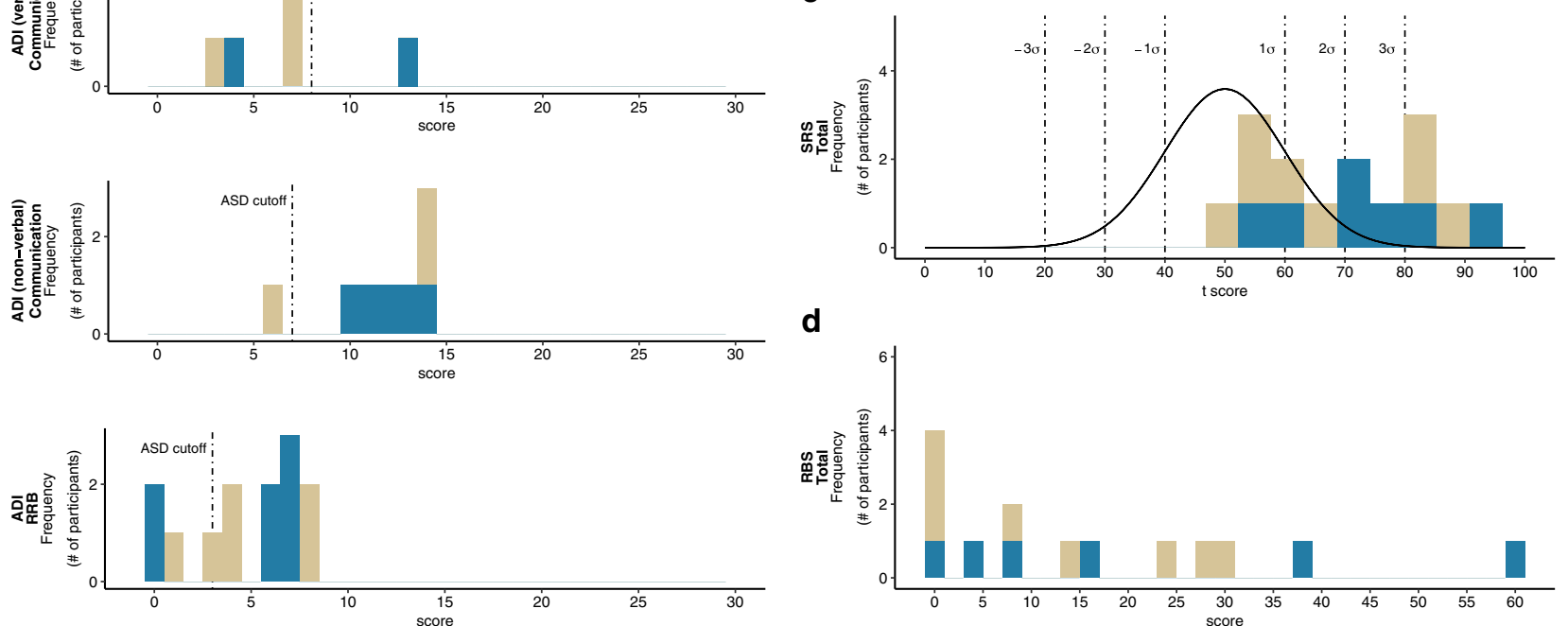

Fig. 3 Psychiatric features. a Frequency histograms for the Autism Diagnostic Interview-Revised (ADI-R) Socialization, Communication, and Restricted/Repetitive Behavior (RRB) domains. Dashed lines represent the diagnostic threshold for ASD for each domain, with scores to the right surpassing the threshold. b Frequency histograms for the Autism Diagnostic Observation Schedule-Second Edition (ADOS-2) comparison score. Evidence levels for ASD are categorized as minimum-to-no evidence, low, moderate, and high evidence. c Frequency histograms for the Social Responsiveness Scale (SRS-2) total score. Total score has a mean of 50 and standard deviation of 10. $\mathbf{d}$ Frequency histograms for the Repetitive Behaviors Scale-Revised (RBS-R). Total scores have a minimum of 0 and maximum of 129. In all plots, higher scores indicate greater deficits. In $\mathbf{c}$, $\mathbf{d}$, distribution of standard scores in typically developing individuals are shown as black lines, together with associated standard deviations (dashed lines). PTV, protein-truncating variant; missense, missense variant or in-frame deletion

indicated that ADOS-2 scores in these participants were impacted by the presence of language delays and repetitive behavior domain symptoms (e.g., sensory interests).

Seven of 13 participants met the diagnostic threshold for autism in all four domains on the ADI-R (two caregivers were not administered the ADI-R to reduce caregiver strain once a diagnosis of ASD was excluded) (Fig. 3a, Additional file 1: Table S2). Examining individual domains, 9 of these 13 participants met or surpassed the cutoff on the Socialization domain, 8 met the cutoff on the Communication domain, 10 met the cutoff on the Restricted and Repetitive Behavior domain, and all individuals who completed the ADI-R met the cutoff on the Abnormality Evident by 36 Months domain.

Results from the SRS-2 $(n=15)$ indicated that $80 \%$ of the cohort had deficits in the Social Awareness, Social Cognition, and Repetitive Behavior domains, 60\% showed deficits in the Social Communication domain, and $47 \%$ in the Social Motivation domain (Fig. 3c). On the RBS-R $(n=14)$, the greatest number of symptoms was reported for Insistence on Sameness (36 symptoms reported across the cohort, with individual participant symptom counts ranging from 0 to 9) and Stereotyped Behavior (37 symptoms reported across the cohort, with individual symptom counts ranging from 0 to 6) (Fig. 3d).

\section{Sensory features}

Sensory responsivity data were collected using the SAND $(n=15)$, and $z$-scores were calculated based on an age-matched sample of typically developing controls recruited as part of ongoing studies at the Seaver Autism Center ( $n=29 ; 26$ females) (Fig. 4). The DDX3X syndrome group displayed significantly more sensory symptoms across symptom domains and sensory modalities compared to the control group (Table 2). Sensory hyperreactivity scores ranged from average to $>3$ SDs above the mean. The majority of participants 

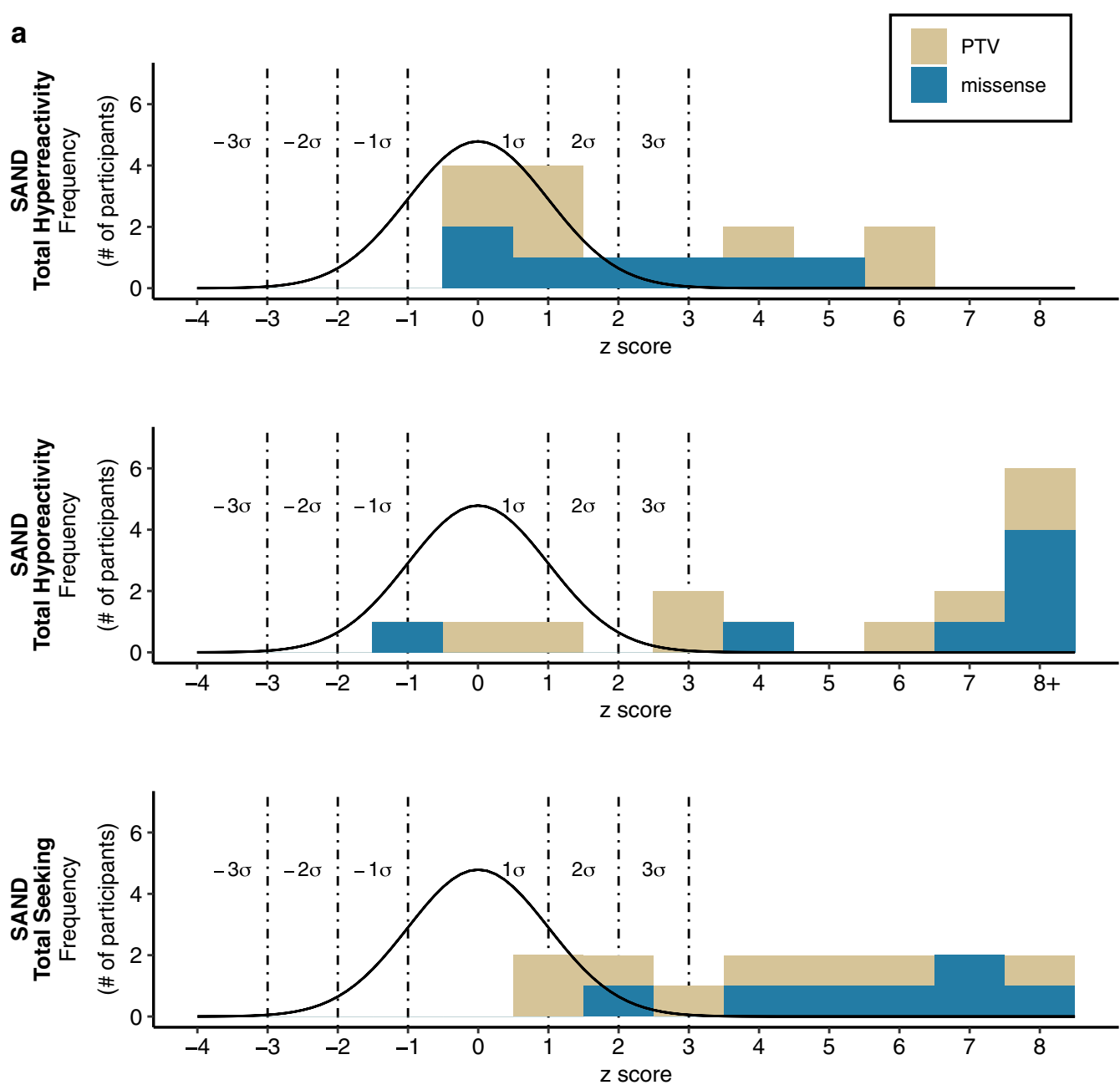

b

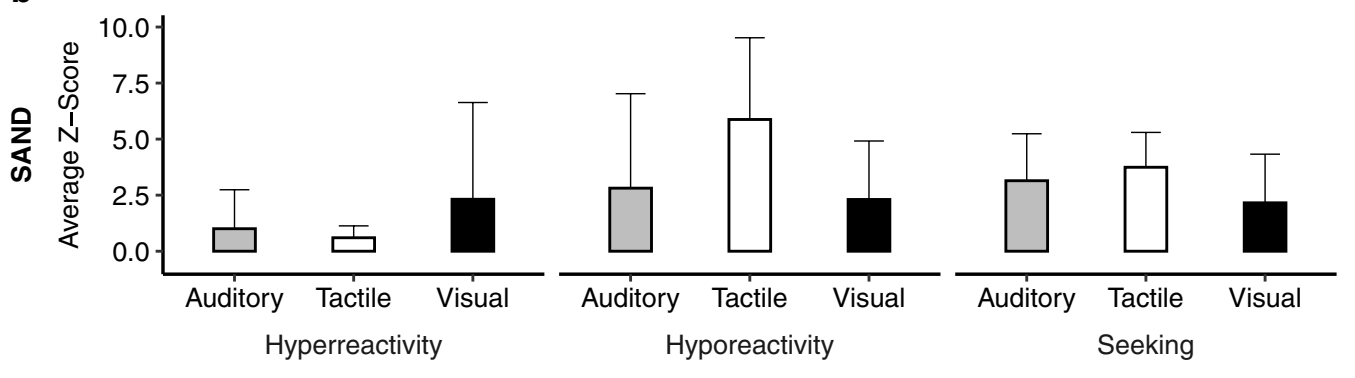

SAND subdomains

Fig. 4 Sensory reactivity. a Frequency histograms for the Sensory Assessment for Neurodevelopmental Disorders (SAND) hyperreactivity, hyporeactivity and sensory seeking domains. Distribution of standard scores in typically developing individuals are shown as black lines, together with associated standard deviations (dashed lines). b Average Z-scores for hyperreactivity, hyporeactivity, and seeking within visual, tactile, and auditory modalities. Z-scores have a mean of 0 where +1 indicates 1 SD above the mean. PTV, protein-truncating variant; missense, missense variant or in-frame deletion

scored $>2$ SDs above the mean in the sensory hyporeactivity domain (12/15) and $>2$ SDs above the mean in the sensory seeking domain $(13 / 15)$. On average, sensory seeking and hyporeactivity were more common than hyperreactivity symptoms. Within sensory modalities, the cohort showed a greater number of tactile symptoms compared to auditory and visual symptoms, although scores were elevated relative to controls in 
all modalities. Tactile hyporeactivity (e.g., high pain threshold) was more commonly observed than visual and auditory hyporeactivity, while visual hyperreactivity was more common than tactile and auditory hyperreactivity.

On the SSP $(n=15)$, total scores indicated definite sensory differences in $40 \%$ of individuals, possible differences in $26.7 \%$ individuals, and typical performance in $33.3 \%$ individuals. The greatest number of reported symptoms was in the Under-responsive/Seeks Sensation subdomain, in which definite sensory differences were reported in 11 individuals.

\section{Behavioral comorbidities}

Domains from the school-age and pre-school versions of the CBCL $(n=14)$ were combined to evaluate additional psychiatric symptomatology (Table 2, Additional file 2: Figure S1-A). Results from the Internalizing domain indicated clinically significant results for $14 \%$ of the cohort and results from the Externalizing domain indicated clinically significant results in $21 \%$ of the cohort. Results from the Aberrant Behavior Checklist indicated that 20\% of the cohort scored in the clinically significant range in the Hyperactivity and Stereotypy domains, 13\% in the Irritability domain, and $7 \%$ in each the Lethargy/Social Withdrawal and Inappropriate Speech domains.

On the Internalizing subdomain of the Maladaptive Behavior domain from the Vineland-3, v-scores ranged from 15 to $22(18.5 \pm 2.2)$ and from 11 to $21(17.5 \pm 2.6)$ on the Externalizing subdomain (Additional file 2: Figure S1-B). Clinical evaluation by the psychiatrist and review of all available assessments indicated that 8 of 15 participants met consensus diagnoses for DSM-5 ADHD, combined type, and 1 for DSM-5 Generalized Anxiety Disorder.

\section{Medical evaluation}

Medical, neurological, and clinical genetic findings are separated by frequency, operationalized as frequent (>50\%), common (20-50\%), and other less frequent findings (<20\%) (Table 3 and Additional file 1: Tables S3-S5).

\section{Frequent findings}

Gait disturbance was ubiquitous (15/15). Gait abnormalities included apraxic, ataxic, or disorganized gait and toe-walking (Tables 3, Additional file 1: Table S3-4). Two individuals required walkers. Hypotonia was present in 14/15 individuals and most often considered mild at the time of assessment. Structural brain changes were present in 12 of 14 participants, with the most common finding being an abnormality of the corpus callosum (9/14), followed by key-hole shaped temporal horns (7/14), enlarged ventricles
Table 3 Common (> 50\%) medical findings

\begin{tabular}{lll}
\hline Feature & Percentage & $\boldsymbol{n}$ \\
\hline Gait abnormalities & 100 & $15 / 15$ \\
Hypotonia & 93 & $14 / 15$ \\
Malar hypoplasia & 93 & $13 / 14$ \\
Flat midface & 93 & $13 / 14$ \\
Structural brain changes & 86 & $12 / 14$ \\
Corpus callosum abnormalities & 64 & $9 / 14$ \\
Key-hole shaped temporal horns & 50 & $7 / 14$ \\
Enlarged ventricles & 43 & $6 / 14$ \\
White matter abnormalities & 43 & $6 / 14$ \\
Vermis abnormalities & 21 & $3 / 14$ \\
Gastrointestinal problems & 80 & $12 / 15$ \\
Constipation & 53 & $8 / 15$ \\
Reflux & 53 & $8 / 15$ \\
Ocular abnormalities & 73 & $11 / 15$ \\
Strabismus & 40 & $6 / 15$ \\
Astigmatism & 33 & $5 / 15$ \\
Myopia & 20 & $3 / 15$ \\
Amblyopia & 13 & $2 / 15$ \\
Hyperopia & 13 & $2 / 15$ \\
Sleep disturbance & 67 & $10 / 15$ \\
Feeding issues & 67 & $10 / 15$ \\
Hyperextensibility & 64 & $9 / 14$ \\
Pigmented skin lesions & 62 & $8 / 13$ \\
Bulbous nose & 57 & $8 / 14$ \\
High arched palate & 57 & $3 / 15$ \\
Recurrent infections & 53 & \\
Otitis media & 40 & \\
Urinary tract & 20 & \\
Respiratory tract & & \\
Pointed chin & 5715 \\
\hline
\end{tabular}

(6/14), white matter abnormalities (6/14), and vermis abnormalities (3/14). One individual had polymicrogyria. Gastrointestinal problems were frequently reported (12/15); constipation was the most common issue $(8 / 15)$ followed by gastroesophageal reflux (7/15). Ocular abnormalities were reported in 11/15 individuals; strabismus was most common $(6 / 15)$ followed by astigmatism (5/15), myopia (3/15), amblyopia (2/15), hyperopia (2/15), and nystagmus (1/15). Sleep disturbance was reported in 10/15 individuals, often characterized by difficulty falling asleep, night wakening, and difficulty falling back asleep. Feeding issues were reported in 10/15 of individuals. The average body mass index (BMI) in our cohort was $17.28(n=14)$, which is slightly below the normal BMI range of 18.5 to 24.9; however, children under ten years old $(n=10)$ had an average BMI of 15.3, which is considered severely underweight, whereas children over ten $(n=4)$ had an 
average BMI of 22.2, which is within the typical BMI range.

Pigmented skin lesions were reported in 8/13 individuals. Recurrent infections were often reported (8/15); the most common was recurrent otitis media (6/15), followed by urinary tract infections (3/15), and respiratory infections (3/15).

Similar dysmorphic features were identified across the cohort $(n=14)$ (Table 3; Fig. 5). Notably, all but one individual had malar hypoplasia and a flat midface. A majority of participants had a high arched palate (57\%) and a bulbous nose $(57 \%)$, while $50 \%$ had a pointed chin. Other dysmorphic features included nail hypoplasia (46\%), wide nasal bridge (43\%), long philtrum (43\%), full cheeks (29\%), and widely spaced teeth (29\%).

\section{Common findings}

Neonatal Intensive Care Unit stays were required for $6 / 15$ individuals (durations ranged from two hours to 15 days, none due to premature birth). Additional neonatal issues gathered through the Pervasive Developmental Disorder Behavior Inventory included difficulty latching/ feeding (11/13), reflux/spit up (4/13), low birth weight (3/13), needed oxygen at birth (3/13), and irritable/ fussy (1/13). Additional pregnancy complications (8/15) included intrauterine growth restriction (3/15), small for gestational age (3/15), gestational diabetes $(2 / 15)$, nuchal cord thickening (2/15), and oligohydramnios $(2 / 15)$. The average birth weight was $2.75 \mathrm{~kg}$ (range: 2.04 to $3.51 \mathrm{~kg}$ ) and the average gestational period was $38+1$ weeks (range: 36-40 weeks). The neurological assessment revealed hypertonia in 5/15 individuals, and four of these individuals also had hypotonia. Hearing abnormalities were reported in three individuals. Precocious puberty was present in 2/10 individuals who were over the age of 5.

\section{Other less frequent findings}

Reported findings include seizures (2/15): one individual had experienced one known seizure and was not currently treated with anti-convulsants and a second individual had a history of staring spells, an abnormal electroencephalogram, and treatment with oxcarbazepine. One individual had a congenital heart defect. Additionally, one participant had both hypothyroidism and obstructive sleep apnea.

\section{Comparisons across variant classes}

An examination of the distribution of neuropsychological profiles as a function of variant class showed evidence for an association of greater severity in clinical phenotype with missense variants/in-frame deletions (Figs. 2, 3, 4).
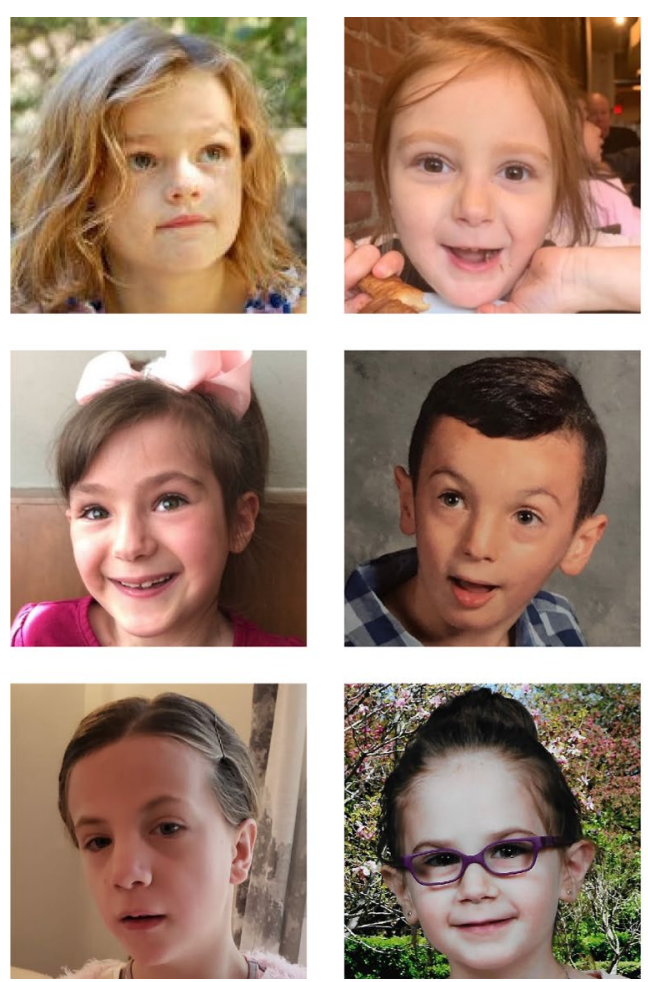
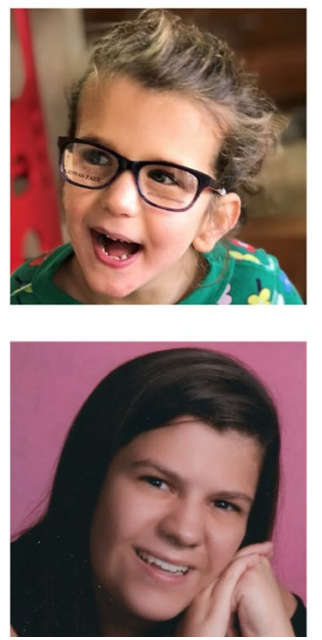
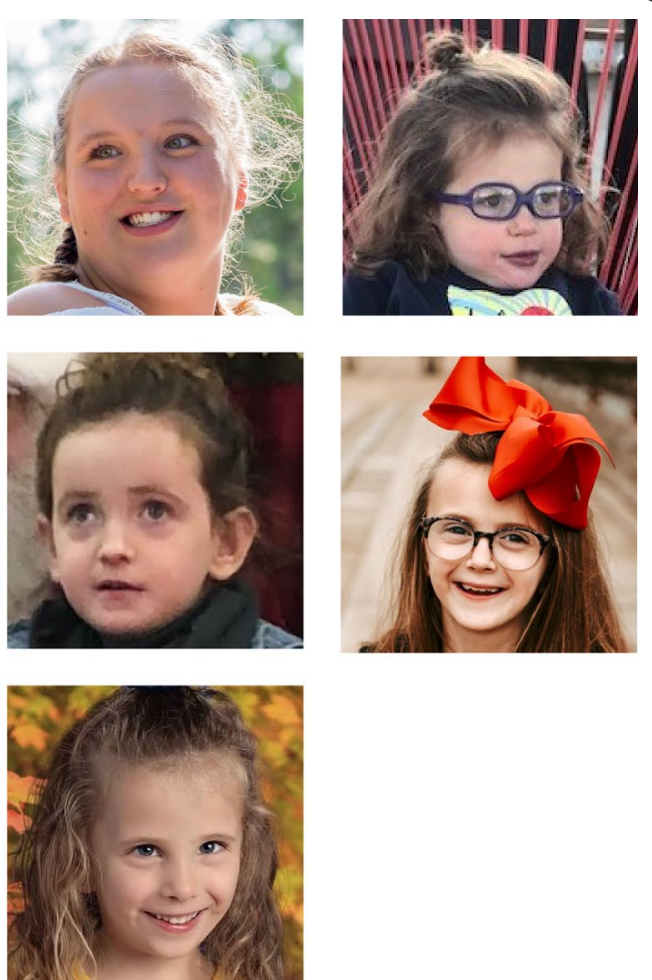

Fig. 5 Photographs of participants 

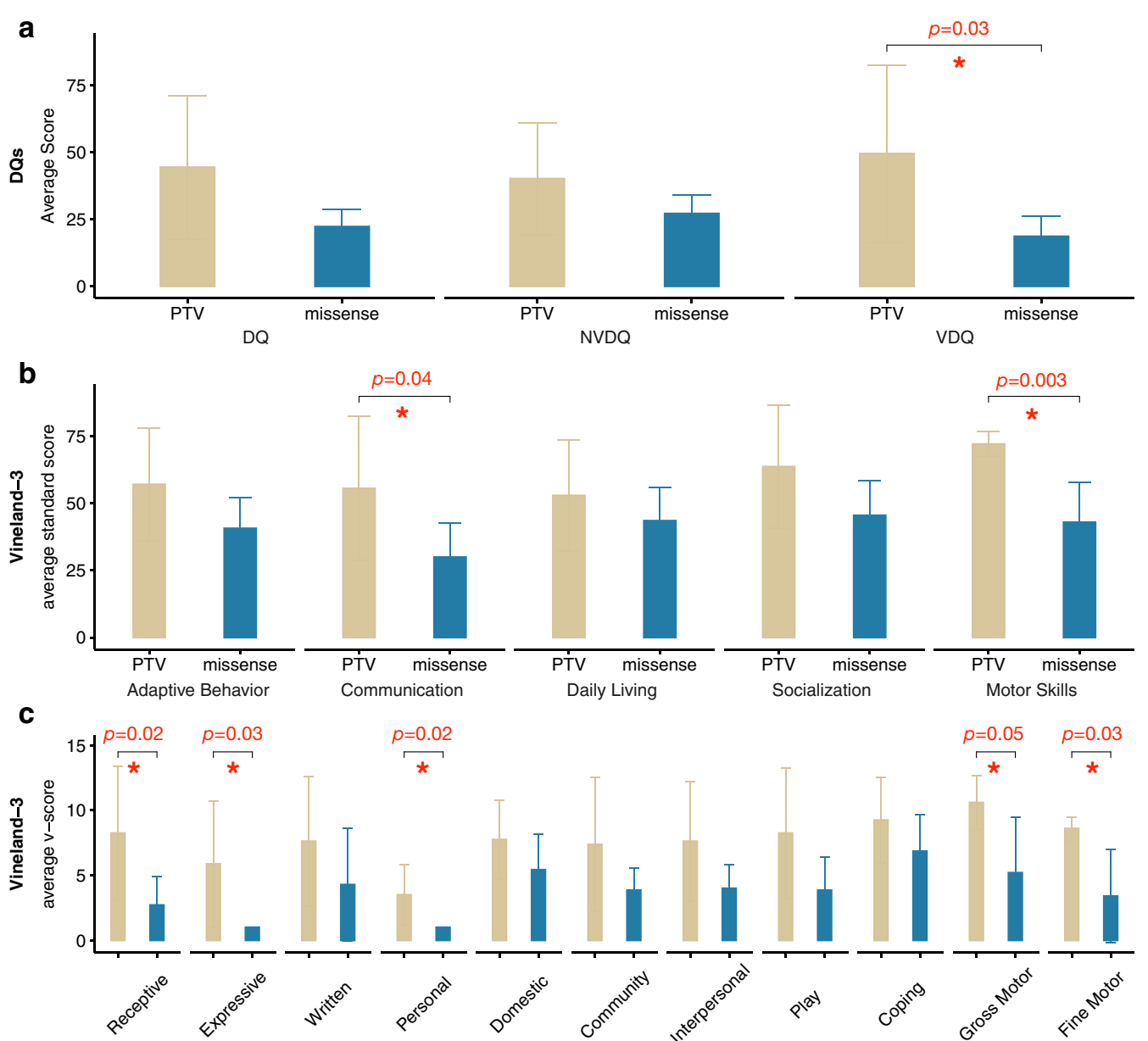

Fig. 6 Phenotypic comparisons across variant classes. a Average scores for full scale DQ, nonverbal DQ, and verbal DQ, comparing the two variant types. b Average Vineland-3 standard scores, comparing the two variant types. c Average Vineland-3 v-scale scores, comparing the two variant types. Independent sample t-tests or chi-square analyses were performed to compare the neurobehavioral profiles across variant types. PTV, protein-truncating variant; missense, missense variant or in-frame deletion

We carried out further, exploratory analyses in a subset of measures, including Vineland-3 scores, DQ, and ASD diagnosis (Fig. 6).

The PTV group had significantly higher verbal DQ scores $(49.5 \pm 33.1, \quad p=0.032)$ when compared to the missense/in-frame deletion group (18.6 \pm 7.4$)$ (Fig. 6a). With regard to adaptive behavior, assessed via the Vineland-3, individuals in the PTV group showed greater skills in multiple areas, when compared to individuals in the missense variant/in-frame deletion group. This was true for the Communication domain $(55.6 \pm 27.0$ versus $30.0 \pm 12.9, \mathrm{p}=0.037)$ [and the Expressive Language subdomain $(5.9 \pm 4.9$ versus $1.0 \pm 0.0, \mathrm{p}=0.025)$, Receptive Language subdomain $(8.38 \pm 5.1$ versus $2.7 \pm 2.2, \mathrm{p}=0.020)]$. Similarly, the PTV group showed significantly higher scores in the
Motor domain $(72.2 \pm 4.5$ versus $43 \pm 14.9, \mathrm{p}=0.003)$ [and the Gross Motor subdomain $(10.6 \pm 2.1$ versus $5.2 \pm 4.3, \mathrm{p}=0.045)$ and the Fine Motor subdomain $(8.6 \pm 0.9$ versus $3.4 \pm 3.6, \mathrm{p}=0.029)]$. Analyses were also performed without data from the participant carrying the c.679+3_679+4delinsTT variant, since functional studies have not been done to definitively classify this variant as a PTV. All statistics stayed significant with the exception of the Vineland-3 Communication domain (Additional file 1: Table S7), however, both the Receptive and Expressive Language subdomains remained significantly different.

A trend was observed for a lower rate of ASD diagnosis in the PTV group (37.5\% versus $85.7 \%, \mathrm{p}=0.057$ ). On multiple measures, Cohen's d effect sizes were $>0.8$ reflecting a large effect (Additional file 1: Table S7). 
Table 4 Comparison with past literature

\begin{tabular}{|c|c|c|c|c|c|}
\hline & Current study & Snijders Blok, 2015 & Wang, 2018 & Beal, 2019 & Lennox, 2020 \\
\hline Sample size (F,M) & 14,1 & 38,0 & 28,0 & 6,0 & 104,3 \\
\hline ID & $80 \% *$ & \multirow{3}{*}{$\begin{array}{l}\text { ID and/or other } \\
\text { delays, 100\% }\end{array}$} & \multirow{3}{*}{$\begin{array}{l}\text { ID and/or other } \\
\text { delays, 100\% }\end{array}$} & $50 \%$ & ND \\
\hline Motor delays & $87 \% *$ & & & \multirow{2}{*}{$\begin{array}{l}\text { Speech/motor } \\
\text { delays, } 67 \%\end{array}$} & ND \\
\hline Speech delays & $93 \% *$ & & & & ND \\
\hline ASD & $60 \% *$ & \multirow{2}{*}{$\begin{array}{c}\text { One or more of ASD, } \\
\text { hyperactivity, and } \\
\text { aggression, 53\% }\end{array}$} & \multirow{2}{*}{$\begin{array}{l}\text { ASD and/or other } \\
\text { behavioral prob- } \\
\text { lems, } 21 \%\end{array}$} & \multirow{2}{*}{$\begin{array}{c}\text { One or more of ASD, } \\
\text { hyperactivity, and } \\
\text { aggression, 33\% }\end{array}$} & $21 \%$ \\
\hline ADHD & $53 \% *$ & & & & $15 \%$ \\
\hline Generalized anxiety disorder & $7 \% *$ & ND & ND & ND & ND \\
\hline Visual motor integration abnormalities & $54 \% *$ & ND & ND & ND & ND \\
\hline Abnormal expressive language $^{1}$ & $80 \% *$ & ND & ND & ND & ND \\
\hline Abnormal receptive language ${ }^{2}$ & $73 \% *$ & ND & ND & ND & ND \\
\hline Sensory symptoms & $100 \% *$ & ND & ND & ND & ND \\
\hline Gait disturbance/movement disorders & $100 \% *$ & $45 \%$ & $61 \%$ & $33 \%$ & ND \\
\hline Hypotonia & $93 \% *$ & $76 \%$ & $68 \%$ & $0 \%$ & $82 \%$ \\
\hline Gastrointestinal abnormalities & $80 \%$ & ND & ND & ND & ND \\
\hline Ocular/vision abnormalities & $73 \% *$ & $34 \%$ & $32 \%$ & $50 \%$ & $36 \%$ \\
\hline Sleep disturbance & $67 \% *$ & ND & ND & $33 \%$ & ND \\
\hline Abnormal brain MRI & $86 \% *$ & $57 \%$ & $90 \%$ & $25 \%$ & $94 \% *$ \\
\hline Corpus callosum abnormalities & $64 \% *$ & $35 \%$ & ND & $25 \%$ & $87 \% *$ \\
\hline Polymicrogyria/cortical dysplasia & $7 \% *$ & $11 \%$ & ND & $0 \%$ & $12 \% *$ \\
\hline Epilepsy/seizures & $13 \%$ & $16 \%$ & ND & $17 \%$ & $20 \%$ \\
\hline Cardiac defects/disease & $7 \%$ & ND & $71 \%^{3}$ & $17 \%$ & $15 \%$ \\
\hline Precocious puberty & $20 \%$ & $13 \%$ & ND & $0 \%$ & $8 \%$ \\
\hline
\end{tabular}

ND, Not Determined, F: Female; M: Male; ID: Intellectual disability; ASD: Autism spectrum disorder; ADHD: Attention-deficit/hyperactivity disorder; MRI: Magnetic resonance imaging

*Prospectively assessed

$1>1.5$ SD below the mean on the Expressive Vocabulary Test, Second Edition or could not complete

$2>1.5$ SD below the mean on the Peabody Picture Vocabular Test, Fourth Edition or could not complete

${ }^{3} 5$ of 7 individuals assessed

\section{Discussion}

Previous studies of DDX3X syndrome are based largely on chart review of medical histories, with only modest direct real-time assessment of the neurobehavioral and psychiatric manifestations. The literature has mainly focused on ID/DD severity, neurological phenotype (e.g., hypotonia, structural brain abnormalities, epilepsy, movement disorders), and medical comorbidities such as precocious puberty, visual/hearing abnormalities and scoliosis $[1,13,15,16]$ (Table 4). The behavioral phenotype has been noted but not described in detail $[1,16]$, and ASD, ADHD, and sensory features have not been directly studied. To address this knowledge gap, in the current study we carried out extensive phenotyping, using prospective neuropsychological, neurological, clinical genetic, and psychiatric assessments in 15 individuals with DDX3X syndrome.
In our cohort, consistent with previous reports, $D D X 3 X$ variants clustered in the helicase ATP-binding domain or the helicase- $C$ domain, with the exception of one variant occurring at the start codon. Our cohort included 4 novel variants (p.Glu285Lys, p.Gln241Hisfs"53, p.Gln308*, and p.Arg292Leu) and 11 that were previously identified. Previous literature has suggested that the Arg326His variant is associated with a more severe clinical outcome [13]. This variant has been reported in the literature four times, and all individuals have polymicrogyria $[1,13]$. Our cohort has a participant with a variant at the same amino acid, Arg326Cys, who also has polymicrogyria, further implicating an association between variants at this location and polymicrogyria. Additionally, individuals with variants at amino acid 415 have been reported to have a more severe clinical presentation. Four individuals have 
been described with variants at this location, 2 with polymicrogyria and 3 with corpus callosum abnormalities and enlarged ventricles $[13,15]$. The participant in our cohort does not have polymicrogyria, however severe developmental delays and medical comorbidities are present.

Cognitive functioning was directly evaluated, and $80 \%$ of our cohort met criteria for a DSM-5 diagnosis of ID. We observed a wide range of cognitive abilities, which appeared to be associated with the participants' variant type, where those with missense variants had lower scores on average. Additionally, participants without ASD had significantly higher DQ scores than participants with ASD. The presence of ADHD did not show an association with DQ scores. Adaptive behavior deficits were present in all participants, indicating that even individuals with higher cognitive ability had challenges applying their skills appropriately in daily life.

Language milestones were significantly delayed, and 5 of 15 individuals were non-verbal at the time of assessment. For those in the cohort who were verbal, both receptive and expressive language skills were significantly below age expectations, with expressive language scores showing greater deficits than receptive language scores. The average age of first words occurred at 2.4 years, more than a year after typically expected. Participants without ASD scored significantly higher on all language assessments compared to individuals with ASD.

In addition, behavioral comorbidities were prospectively studied for the first time. DSM- 5 diagnoses of ASD were present in $60 \%$ of the cohort, and many of the participants who did not meet full ASD criteria exhibited ASD traits such as repetitive behaviors and sensory symptoms. Over half of individuals presented with clinically significant hyperactivity and attention problems and met criteria for ADHD, combined type. Frustration intolerance was also common.

Results from sensory assessments indicated higher rates of sensory hyporeactivity and sensory seeking, compared to typically developing counterparts. Sensory hyporeactivity has been implicated in other genetic syndromes associated with ASD such as Phelan-McDermid syndrome and ADNP syndrome $[45,46]$ and is often associated with high pain tolerance.

Common medical comorbidities included gastrointestinal difficulties, such as constipation and reflux, and recurrent common infections, such as ear, urinary tract, and respiratory tract infections. The ocular phenotype was extended to include features such as astigmatism, myopia, amblyopia, nystagmus, and hyperopia, in addition to strabismus. We replicated evidence of brain abnormalities, microcephaly, and a range of gait abnormalities for those affected by DDX3X syndrome.

Recent analyses of the DDX3X syndrome phenotype provided initial evidence for the association of distinct classes of genetic variants with the severity of phenotypes [13]. When we compared phenotypes of individuals with PTVs to those with missense variants or in-frame deletions, we confirmed that individuals with missense variants, on average, demonstrated more severe clinical phenotypes, including lower intellectual, language, and adaptive functioning. We performed analyses with and without the participant with polymicrogyria and still see significant differences between the missense and PTV groups; hence the presence of polymicrogyria, by itself, does not appear to be a simple way of defining the most severe mutations (Additional file 1: Table S7). A significant limitation for these analyses is that, with the current sample size, we did not correct for multiple testing. However, for many findings, the Cohen's d effect sizes were $>0.8$, indicating that larger sample sizes will continue to show significant differences.

Prospective studies are both expensive and time-consuming, but can have important advantages compared to medical record review. First, such studies can apply gold standard diagnostic instruments which may not be used in all typical clinical settings. In addition, all assessments can be done by a single integrated team, which provides more consistent phenotyping and opportunities for consensus diagnoses. From our analyses, a significant proportion of individuals with DDX3X syndrome have definitive diagnoses of ASD and ADHD. Another advantage of prospective approaches is that subclinical assessments and biomarker determination can be part of the phenotyping. In the current report, we include measures such as SRS-2 and a novel sensory biomarker assessment (SAND), demonstrating, for the first time, definitive sensory changes are present in DDX3X syndrome. Finally, in prospective, integrated studies, all items and results can be shared via de-identified databases, subject to consent and appropriate compliance review, without requiring additional consent from participants.

\section{Limitations}

The main limitation of our study is the small sample size. Additionally, without functional studies on each of the variants in our cohort we were unable to group variants into functional categories (e.g., PTVs, hypomorphic missense, or dominant negative missense variants) [13]. We instead grouped them into two classes (i.e., PTVs, and missense/in-frame deletions, with the latter 
category including both hypomorphic and dominant negative alleles).

\section{Conclusions}

DDX3X syndrome is emerging as a major cause of NDDs in girls. This study uniquely explored the clinical and neuropsychiatric phenotype of individuals with DDX3X syndrome by using prospective and in-person analyses. Comprehensive clinician-administered evaluations and standardized diagnostic and neuropsychological assessments were administered to characterize the neuropsychiatric dimensions of DDX3X syndrome, including the prevalence of ASD, and to more clearly define language and adaptive functioning in these individuals. Our findings offer a deeper understanding of the behavioral, medical, and developmental challenges that children and adolescents with DDX3X syndrome experience, while informing clinical guidance and potential interventions. As research focused on delineating the clinical phenotypes and natural history of this syndrome continues, more tailored, comprehensive and developmentallyinformed assessment and treatment approaches will emerge. These resources will, in turn, support caregivers and clinicians in providing improved care, treatment, and clinical guidance for the conditions and challenges that affect those with DDX3X syndrome.

Looking ahead, genotype-phenotype correlations in DDX3X syndrome will benefit from robust and uniform phenotypic approaches. In a prospective research setting, specific variants or cases of interest can be oversampled, which allows exploration of more rare events and/or emergent events that require, or demand, further analyses. The role of potential dominant negative variants in phenotypic expression will be part of our future studies. In addition, the prospective studies reported here can form the basis for longitudinal studies on DDX3X syndrome.

\footnotetext{
Abbreviations

ADHD: Attention Deficit/Hyperactivity Disorder; ADI: Autism Diagnostic Interview; ADI-R: Autism Diagnostic Interview-Revised; ADOS-2: Autism Diagnostic Observation Schedule, Second Edition; ASD: Autism Spectrum Disorder; BMI: Body Mass Index; CBCL: Achenbach Child Behavior Checklist; DAS-II: Differential Ability Scales, Second Edition; DCDQ: Developmental Coordination Disorder Questionnaire; DD: Developmental Delay; DQ: Developmental Quotient; DSM-5: Diagnostic and Statistical Manual, Fifth Edition; ID: Intellectual Disability; IQ: Intellectual Quotient; MRI: Magnetic Resonance Imaging; NDDs: Neurodevelopmental Disorders; PTV: Protein-Truncating Variant; RBS-R: Repetitive Behavior Scale-Revised; SAND: Sensory Assessment for Neurodevelopmental Disorders; SRS-2: Social Responsiveness Scale, Second Edition; SSP: Short Sensory Profile; Vineland-3: Vineland Adaptive Behavior Scales, Third Edition; VMI-6: Beery-Buktenica Developmental Test of Visual-Motor Integration, Sixth Edition.
}

\section{Supplementary Information}

The online version contains supplementary material available at https://doi. org/10.1186/s13229-021-00431-z.

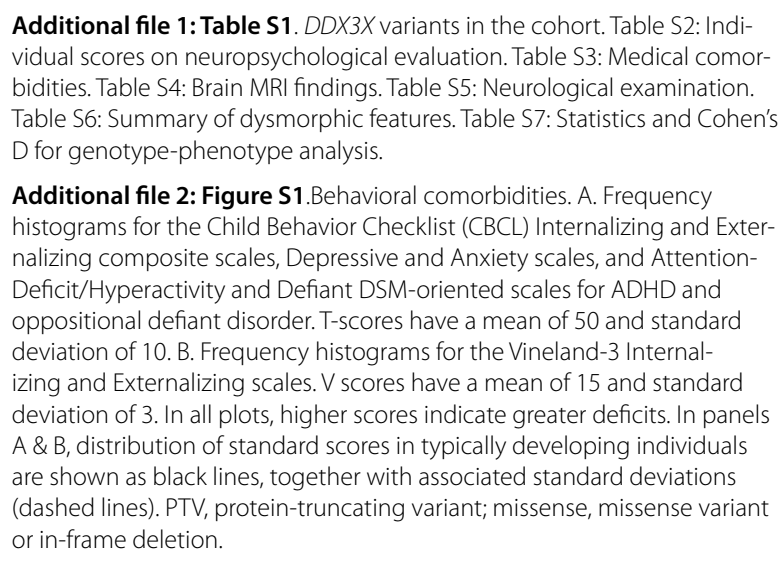
vidual scores on neuropsychological evaluation. Table S3: Medical comorbidities. Table S4: Brain MRI findings. Table S5: Neurological examination. Table S6: Summary of dysmorphic features. Table S7: Statistics and Cohen's D for genotype-phenotype analysis.

Additional file 2: Figure S1.Behavioral comorbidities. A. Frequency histograms for the Child Behavior Checklist (CBCL) Internalizing and Externalizing composite scales, Depressive and Anxiety scales, and AttentionDeficit/Hyperactivity and Defiant DSM-oriented scales for ADHD and oppositional defiant disorder. T-scores have a mean of 50 and standard deviation of 10. B. Frequency histograms for the Vineland-3 Internalizing and Externalizing scales. $V$ scores have a mean of 15 and standard deviation of 3 . In all plots, higher scores indicate greater deficits. In panels $A \& B$, distribution of standard scores in typically developing individuals are shown as black lines, together with associated standard deviations (dashed lines). PTV, protein-truncating variant; missense, missense variant or in-frame deletion.

\section{Acknowledgements}

The Seaver Autism Center research team would like to thank all of the families affected by DDX3X syndrome, not only for their time, patience, and generous efforts in participating in our research studies, but also for their dedication and commitment to the larger scientific and research community.

\section{Authors' contributions}

Conception and design of study: DEG, JDB, PMS, AKol; Acquisition of data: $\mathrm{DH}, J \mathrm{Z}, \mathrm{IGK}, J \mathrm{HF}, \mathrm{YF}, \mathrm{RL}, \mathrm{CL}, \mathrm{PMS}, \mathrm{DEG}$; Analysis and/or Interpretation: DEG, TL, JHF, LT, CL, PB, JDB, PMS; Drafting the manuscript: DEG, LT, TL SG, JDB; Revising the manuscript for critically for important intellectual content: $L T, T L, J H F, S D R$, AKos, AKol, JDB, PMS, DEG; Approval of the version of the manuscript to be published: LT, TL, SG, DH, JZ, IGK, JHF, YF, RL, CL, PB, EF, MSB, SDR, AKos, AKol, JDB, PMS, DEG. All authors read and approved the final manuscript.

\section{Funding}

Funding was provided by the Beatrice and Samuel A. Seaver Foundation. The study sponsors had no role in the study design; in the collection, analysis and interpretation of the data; in the writing of the report; and in the decision to submit the paper for publication. SDR is a Fascitelli Research Scholar. MSB is a Seaver Faculty Scholar.

\section{Availability of data and materials}

The majority of the dataset used during the current study is included in this published article and Supplementary File. The remainder of the dataset is available from the corresponding author on reasonable request and may require ethics review.

\section{Declarations}

\section{Ethics approval and consent to participate}

Parents or legal guardians of all participants in this study provided informed consent prior to study participation. This study was approved by the Mount Sinai Institutional Review Board, Program for the Protection of Human Subjects (Study ID: 98-436).

\section{Consent for publication}

Additional consent for publication of data was obtained from all parents and guardians. Specific consent for use of all photographs was also obtained.

\section{Competing interests}

A. Kolevzon receives research support from AMO Pharma and consults to Ovid Therapeutics, Acadia, and Sema4. PMS is the inventor of the SAND, which is licensed by Mount Sinai to Stoelting Co. No other competing interests to declare. 


\section{Author details}

${ }^{1}$ Seaver Autism Center for Research and Treatment, Icahn School of Medicine at Mount Sinai, 1425 Madison Avenue, Box 1230, New York, NY 10029, USA. ${ }^{2}$ Department of Psychiatry, Icahn School of Medicine at Mount Sinai, New York, NY 10029, USA. ${ }^{3}$ Department of Genetics and Genomic Sciences, Icahn School of Medicine at Mount Sinai, New York, NY 10029, USA. ${ }^{4}$ Department of Pediatrics, Icahn School of Medicine at Mount Sinai, New York, NY 10029, USA. ${ }^{5}$ Department of Radiology, Icahn School of Medicine at Mount Sinai, New York, NY 10029, USA. ${ }^{6}$ Icahn Institute for Data Science and Genomic Technology, Icahn School of Medicine at Mount Sinai, New York, NY 10029, USA. ${ }^{7}$ Pamela Sklar Division of Psychiatric Genomics, Icahn School of Medicine at Mount Sinai, New York, NY 10029, USA. ${ }^{8}$ The Mindich Child Health and Development Institute, Icahn School of Medicine at Mount Sinai, New York, NY 10029, USA. ${ }^{9}$ Friedman Brain Institute, Icahn School of Medicine at Mount Sinai, New York, NY, USA. ${ }^{10}$ Department of Neuroscience, Icahn School of Medicine at Mount Sinai, New York, NY 10029, USA. ${ }^{11}$ Division of Tics, OCD, and Related Disorders, Icahn School of Medicine at Mount Sinai, New York, NY 10029, USA

\section{Received: 26 October 2020 Accepted: 1 March 2021} Published online: 16 May 2021

\section{References}

1. Snijders Blok L, Madsen E, Juusola J, Gilissen C, Baralle D, Reijnders MR, et al. Mutations in DDX3X are a common cause of unexplained intellectual disability with gender-specific effects on Wnt signaling. Am J Hum Genet. 2015;97(2):343-52.

2. Deciphering Developmental Disorders Study. Prevalence and architecture of de novo mutations in developmental disorders. Nature. 2017;542(7642):433-8.

3. Ruzzo EK, Perez-Cano L, Jung JY, Wang LK, Kashef-Haghighi D, Hartl C, et al. Inherited and de novo genetic risk for autism impacts shared networks. Cell. 2019:178(4):850-66.e26

4. Yuen RK, Merico D, Bookman MJLH, Thiruvahindrapuram B, Patel RV, et al. Whole genome sequencing resource identifies 18 new candidate genes for autism spectrum disorder. Nat Neurosci. 2017;20(4):602-11.

5. Takata A, Miyake N, Tsurusaki Y, Fukai R, Miyatake S, Koshimizu E, et al. Integrative analyses of de novo mutations provide deeper biological insights into autism spectrum disorder. Cell Rep. 2018;22(3):734-47.

6. Iossifov I, O'Roak BJ, Sanders SJ, Ronemus M, Krumm N, Levy D, et al. The contribution of de novo coding mutations to autism spectrum disorder. Nature. 2014;515(7526):216-21.

7. Sharma D, Jankowsky E. The Ded1/DDX3 subfamily of DEAD-box RNA helicases. Crit Rev Biochem Mol Biol. 2014:49(4):343-60.

8. Lai MC, Lee YH, Tarn WY. The DEAD-box RNA helicase DDX3 associates with export messenger ribonucleoproteins as well as tip-associated protein and participates in translational control. Mol Biol Cell. 2008;19(9):3847-58.

9. Valiente-Echeverria F, Hermoso MA, Soto-Rifo R. RNA helicase DDX3: at the crossroad of viral replication and antiviral immunity. Rev Med Virol. 2015;25(5):286-99.

10. Szappanos D, Tschismarov R, Perlot T, Westermayer S, Fischer K, Platanitis $E$, et al. The RNA helicase DDX3X is an essential mediator of innate antimicrobial immunity. PLoS Pathog. 2018;14(11):e1007397.

11. Soto-Rifo R, Ohlmann T. The role of the DEAD-box RNA helicase DDX3 in mRNA metabolism. Wiley Interdiscip Rev RNA. 2013;4(4):369-85.

12. Chen HH, Yu HI, Tarn WY. DDX3 modulates neurite development via translationally activating an RNA regulon involved in Rac1 activation. J Neurosci. 2016:36(38):9792-804

13. Lennox $A L$, Hoye ML, Jiang $R$, Johnson-Kerner BL, Suit LA, Venkataramanan S, et al. Pathogenic DDX3X mutations impair RNA metabolism and neurogenesis during fetal cortical development. Neuron. 2020;106(3):404-20.

14. Yuen RKC, Merico D, Cao H, Pellecchia G, Alipanahi B, Thiruvahindrapuram $B$, et al. Genome-wide characteristics of de novo mutations in autism. npj Genom Med. 2016;1(1):16027.
15. Wang X, Posey JE, Rosenfeld JA, Bacino CA, Scaglia F, Immken L, et al. Phenotypic expansion in DDX3X-a common cause of intellectual disability in females. Ann Clin Transl Neurol. 2018;5(10):1277-85.

16. Beal B, Hayes I, McGaughran J, Amor DJ, Miteff C, Jackson V, et al. Expansion of phenotype of DDX3X syndrome: six new cases. Clin Dysmorphol. 2019;28(4):169-74.

17. Chen CY, Chan CH, Chen CM, Tsai YS, Tsai TY, Wu Lee YH, et al. Targeted inactivation of murine $\mathrm{Ddx} 3 \mathrm{x}$ : essential roles of $\mathrm{Ddx} 3 \mathrm{x}$ in placentation and embryogenesis. Hum Mol Genet. 2016;25(14):2905-22.

18. Garieri M, Stamoulis G, Blanc X, Falconnet E, Ribaux P, Borel C, et al. Extensive cellular heterogeneity of $X$ inactivation revealed by singlecell allele-specific expression in human fibroblasts. Proc Natl Acad Sci. 2018;115(51):13015-20.

19. Kellaris G, Khan K, Baig SM, Tsai IC, Zamora FM, Ruggieri P, et al. A hypomorphic inherited pathogenic variant in DDX3X causes male intellectual disability with additional neurodevelopmental and neurodegenerative features. Hum Genomics. 2018;12(1):11.

20. Nicola P, Blackburn PR, Rasmussen KJ, Bertsch NL, Klee EW, Hasadsri $L$, et al. De novo DDX3X missense variants in males appear viable and contribute to syndromic intellectual disability. Am J Med Genet A. 2019;179(4):570-8.

21. Richards S, Aziz N, Bale S, Bick D, Das S, Gastier-Foster J, et al. Standards and guidelines for the interpretation of sequence variants: a joint consensus recommendation of the American College of Medical Genetics and Genomics and the Association for Molecular Pathology. Genet Med. 2015;17(5):405-24.

22. Roid GH, Pomplun M. The stanford-binet intelligence scales. New York: The Guilford Press; 2012.

23. Elliot C. Differential ability scales—second edition (DAS-II). San Antonio: Psychological Corporation; 2007.

24. Mullen EM. Mullen scales of early learning: AGS Circle Pines, MN; 1995.

25. Hill TL, Saulnier CA, Cicchetti D, Gray SAO, Carter AS. Vineland III. In: Volkmar FR, editor. Encyclopedia of autism spectrum disorders. New York: Springer; 2017. p. 1-4.

26. Association AP. Diagnostic and statistical manual of mental disorders (DSM- $\left.5^{\circledR}\right)$ : American Psychiatric Pub; 2013.

27. Lord C, Rutter M, Le Couteur A. Autism Diagnostic Interview-Revised: a revised version of a diagnostic interview for caregivers of individuals with possible pervasive developmental disorders. J Autism Dev Disord. 1994;24(5):659-85

28. Thurm A, Manwaring SS, Luckenbaugh DA, Lord C, Swedo SE. Patterns of skill attainment and loss in young children with autism. Dev Psychopathol. 2014;26(1):203-14.

29. Beery K. Beery VMI administration, scoring, and teaching manual. Bloomington: Pearson; 2010.

30. Wilson BN, Crawford SG, Green D, Roberts G, Aylott A, Kaplan BJ. Psychometric properties of the revised Developmental Coordination Disorder Questionnaire. Phys Occup Ther Pediatr. 2009;29(2):182-202.

31. Williams KT. Expressive vocabulary test second edition $\left(E V T^{\mathrm{TM}} 2\right)$. J Am Acad Child Adolesc Psychiatry. 1997;42:864-72.

32. Dunn L, Dunn D. Peabody picture vocabulary test. 4th ed. Bloomington: NCS Pearson; 2007.

33. Fenson L. MacArthur-Bates communicative development inventories. Baltimore: Paul H. Brookes Publishing Company; 2007.

34. Lord C, Rutter M, DiLavore P, Risi S, Gotham K, Bishop S. Autism diagnostic observation schedule-2nd edition (ADOS-2). Los Angeles: Western Psychological Corporation; 2012.

35. Constantino JN, Gruber CP. Social Responsiveness Scale Second Edition (SRS-2): Manual: Western Psychological Services (WPS); 2012.

36. Lam KS, Aman MG. The Repetitive Behavior Scale-Revised: independent validation in individuals with autism spectrum disorders. J Autism Dev Disord. 2007;37(5):855-66.

37. Siper PM, Kolevzon A, Wang AT, Buxbaum JD, Tavassoli T. A clinicianadministered observation and corresponding caregiver interview capturing DSM-5 sensory reactivity symptoms in children with ASD. Autism Res. 2017;10(6):1133-40. 
38. Dunn W, Westman K. The sensory profile: the performance of a national sample of children without disabilities. Am J Occup Ther 1997;51(1):25-34

39. Achenbach TM. The Child Behavior Checklist and related instruments. The use of psychological testing for treatment planning and outcomes assessment. 2nd ed. Mahwah: Lawrence Erlbaum Associates Publishers; 1999. p. 429-66.

40. Aman MG. Aberrant behavior checklist. In: Volkmar FR, editor. Encyclopedia of autism spectrum disorders. New York: Springer; 2013. p. 10-7.

41. Aman MG, Singh NN. Aberrant behavior checklist: Manual: Slosson East Aurora; 1986

42. Aman MG, Singh N. Aberrant behavior checklist. Community (ABC): Slosson Educational Publications; 1994.

43. Aman MG, Singh NN, Stewart AW, Field CJ. The aberrant behavior checklist: a behavior rating scale for the assessment of treatment effects. Am J Ment Defic. 1985
44. Cohen I, Sudhalter V. Pervasive Developmental Disorder Behavior Inventory (PDDBI-C). NYS Institute for Basic Research in Developmental Disabilities. 1999

45. Van Dijck A, Vulto-van Silfhout AT, Cappuyns E, van der Werf IM, Mancini GM, Tzschach A, et al. Clinical presentation of a complex neurodevelopmental disorder caused by mutations in ADNP. Biol Psychiatry. 2019:85(4):287-97.

46. Mieses AM, Tavassoli T, Li E, Soorya L, Lurie S, Wang AT, et al. Brief report: sensory reactivity in children with Phelan-Mcdermid syndrome. J Autism Dev Disord. 2016;46(7):2508-13.

\section{Publisher's Note}

Springer Nature remains neutral with regard to jurisdictional claims in published maps and institutional affiliations.
Ready to submit your research? Choose BMC and benefit from:

- fast, convenient online submission

- thorough peer review by experienced researchers in your field

- rapid publication on acceptance

- support for research data, including large and complex data types

- gold Open Access which fosters wider collaboration and increased citations

- maximum visibility for your research: over $100 \mathrm{M}$ website views per year

At BMC, research is always in progress.

Learn more biomedcentral.com/submissions 